\title{
Prospects for studying vacuum polarisation using dipole and synchrotron radiation
}

\author{
Anton Ilderton $^{1, \dagger}$ and Mattias Marklund ${ }^{1}$ \\ ${ }^{1}$ Department of Physics, Chalmers University of Technology, SE-41296 Gothenburg, Sweden
}

(Received 1 January 2016; revised 3 February 2016; accepted 4 February 2016)

The measurement of vacuum polarisation effects, in particular vacuum birefringence, using combined optical and X-ray laser pulses are now actively pursued. Here we briefly examine the feasibility of two alternative set-ups. The first utilises an alternative target, namely a converging dipole pulse, and the second uses an alternative probe, namely the synchrotron-like emission from highly energetic particles, themselves interacting with a laser pulse. The latter set-up has been proposed for experiments at ELI-NP.

\section{Introduction}

Light-by-light scattering is a purely quantum effect (Halpern 1933; Euler \& Kockel 1935; Heisenberg \& Euler 1936) which contributes to e.g. the electron magnetic moment, the Lamb shift and Delbrück scattering. In these cases virtual, or both virtual and real, photons are involved, while light-by-light scattering of only real photons has not yet been observed.

While the word 'scattering' suggests momentum change, one manifestation of light-by-light effects is the near-forward scattering of photons with changes to internal degrees-of-freedom, i.e. helicity (or polarisation). Consider the collision of two linearly polarised laser pulses, the first a high-intensity optical pulse, the 'target', the second a low-intensity X-ray pulse, the 'probe' (Heinzl et al. 2006). Due to the separation in energy scales the probe beam essentially scatters forward, but quantum effects can still cause probe photons to change helicity state. This manifests macroscopically as a slight ellipticity in the probe beam and is hence known as 'vacuum birefringence' in analogy to the ellipticity induced in a beam of light passing through a birefringent crystal (Toll 1952). Indeed, many phenomena in nonlinear optics have purely photonic analogues, see Di Piazza, Hatsagortsyan \& Keitel (2006), Heinzl et al. (2006), Marklund \& Shukla (2006), King, Di Piazza \& Keitel (2010), Kim \& Lee (2011), Gies, Karbstein \& Seegert (2013) and Gies, Karbstein \& Seegert (2015).

The measurement of vacuum birefringence has been selected as a flagship experiment by the HiBEF consortium at DESY (HIBEF 2015; Schlenvoigt et al. 2016), following the proposal in Heinzl et al. (2006). For a recent review of the theory behind this topic, see King \& Heinzl (2015) and for a detailed review of the current experimental status, see Schlenvoigt et al. (2016).

$†$ Email address for correspondence: anton.ilderton@chalmers.se 
In this paper we will investigate two alternative, but related, set-ups which have been suggested for measuring light-by-light effects with real photons. Our goal is simply to obtain a very rough idea of how promising these two alternative schemes are: if they seem promising, the calculations presented here can be refined. The first set-up replaces the intense optical laser above with an alternative target, namely an optimally focused 'dipole pulse' (Gonoskov et al. 2012). The second set-up retains the intense optical pulse as target, but replaces the above X-ray probe with high-energy photons (gamma rays) emitted as synchrotron radiation from a laser-particle collision. This is the proposed set-up for measuring helicity-changing processes at the ELI-NP facility in Romania (ELI 2014; Nakamiya et al. 2015).

This paper is organised as follows. In $\S 2$ we describe our approach. In $\S 3$ we consider the alternative target set-up. In $\S 4$ we consider the alternative probe and describe the proposed experimental implementation of such a set-up at ELI-NP. We conclude in $\S 5$.

\section{Helicity flip in background fields}

Recall the standard optics result for the polarisation ellipticity $\delta$ induced in a beam of light, frequency $\omega^{\prime}$, passing through a birefringent medium of length $d$, refractive indices $\left\{n_{+}, n_{-}\right\}$:

$$
\delta=\frac{1}{2}\left(n_{+}-n_{-}\right) \omega^{\prime} d .
$$

The quantum vacuum exposed to a strong field effectively develops 'vacuum refractive indices' which arise through the nonlinearity of the Euler-Heisenberg action (Euler \& Kockel 1935; Heisenberg \& Euler 1936), and can be calculated using the photon polarisation tensor. In the limit that the strong field is a constant, homogeneous crossed field of strength $E$, a counter-propagating probe sees the indices (Toll 1952; Narozhny 1969)

$$
n_{ \pm}=1+\frac{\alpha}{45 \pi}(11 \pm 3) \frac{E^{2}}{E_{S}^{2}},
$$

where $E_{S}=m^{2} / e \simeq 10^{18} \mathrm{~V} \mathrm{~m}^{-1}$ is the Sauter-Schwinger field. Inserting (2.2) into (2.1) we obtain the ellipticity induced in the probe as

$$
\delta \rightarrow \frac{\alpha}{15 \pi} \frac{E^{2}}{E_{S}^{2}} \omega^{\prime} d .
$$

This macroscopic beam ellipticity induced by quantum effects is 'vacuum birefringence'; the microscopic physics underlying it is as follows.

Consider a probe photon, momentum $l_{\mu} \equiv \omega^{\prime} \hat{l}_{\mu}$, frequency $\omega^{\prime}$ and helicity state described by $\epsilon_{\mu}$. The photon passes through a strong background field $F_{\mu \nu}$ with typical frequency scale much smaller than $\omega^{\prime}$ (as would be the case for an X-ray probe of an optical laser). In this case scattering becomes essentially forward. The probability $\mathbb{P}$ that the probe photon flips to its opposite helicity state $\epsilon_{\mu}^{\prime}$ may be written $\mathbb{P}_{\text {flip }}=|\mathbb{T}|^{2}$, where the amplitude $\mathbb{T}$ can be approximated by a line integral over the classical (i.e. straight line) trajectory of the photon (Dinu et al. 2014a), here parameterised with time $t$ :

$$
\mathbb{T}=\frac{\alpha}{30} \frac{\omega^{\prime}}{E_{S}^{2}} \int \mathrm{d} t\left(\bar{\epsilon}_{\mu}^{\prime} F^{\mu \nu} \hat{l}_{\nu}\right)\left(\epsilon_{\sigma} F^{\sigma \rho} \hat{l}_{\rho}\right) .
$$


$F_{\mu \nu}$ is evaluated on the photon trajectory. The probability is maximised when the field and probe polarisations can be chosen to lie at a relative angle of $45^{\circ}$. $\mathbb{T}$ then reduces to

$$
\mathbb{T}=\frac{\alpha}{60} \frac{\omega^{\prime}}{E_{S}^{2}} \int \mathrm{d} t \hat{l}_{\mu} T^{\mu \nu} \hat{l}_{\nu},
$$

where $T_{\mu \nu}$ is the background field energy-momentum tensor; thus we can interpret (2.5) as simply being proportional to an integrated energy density (an intensity) seen by the probe as it passes through the target (Dinu et al. 2014a). That this is an integrated, rather than peak, variable will be important below.

As detailed in Dinu et al. (2014b), the flip probability $\mathbb{P}$ is directly related to the ellipticity $\delta^{2}$ : hence (2.4) is most easily interpreted as a quantum field theory generalisation of the classical result (2.1), which goes beyond (2.3) as it allows us to consider arbitrary field strengths and shapes (on the usual provisos that the field strength is not of Schwinger scale and the invariant, centre of mass (c.o.m.), frequency scales do not exceed the electron mass).

There are several effects which we do not include in this first investigation. No depletion of the background field is accounted for, nor do we account for probe scattering (Lundstrom et al. 2006; King \& Keitel 2012; Karbstein et al. 2015), for an investigation of which in vacuum birefringence, see Karbstein et al. (2015). We also restrict our attention to single photon probes; beam-like probes can be accounted for using a straightforward extension of the formalism used here (Dinu et al. 2014a; Torgrimsson 2015). In summary, our current approximation gives a good estimate for the on-axis birefringence signal. (Note though that in experiments with either laser or magnetic fields scattered photon signals may be easier to detect than on-axis signals, due to lower backgrounds (Gies et al. 2013; Karbstein 2015; Karbstein et al. 2015).)

\subsection{Conventions and notation}

The helicity-state vectors for a photon of momentum $l_{\mu}$ are

$$
\epsilon_{ \pm}^{\mu}=\frac{1}{\sqrt{2}}\left(\epsilon_{1}^{\mu} \pm \mathrm{i} \epsilon_{2}^{\mu}\right),
$$

where, using Coulomb gauge,

$$
\begin{gathered}
l^{\mu}=\omega^{\prime}(1, \sin \theta \cos \phi, \sin \theta \sin \phi, \cos \phi), \\
\epsilon_{1}^{\mu}=(0, \cos \theta \cos \phi, \cos \theta \sin \phi,-\sin \phi), \\
\epsilon_{2}^{\mu}=(0,-\sin \phi, \cos \phi, 0) .
\end{gathered}
$$

\section{Dipole pulse targets}

Dipole pulses are exact, singularity free, optimally focussed, finite-energy solutions of Maxwell's equations in a vacuum (Gonoskov et al. 2012). In an 'e-dipole' pulse the electric field dominates over the magnetic field in the focus, and provides optimal conditions for pair production via the non-perturbative Sauter-Schwinger mechanism (Gonoskov et al. 2013, 2014). In an ' $h$-dipole' pulse, the magnetic field dominates and one might ask whether the optimal focussing amplifies the helicity-flip probability. To investigate this we consider replacing the intense beam in the vacuum birefringence experiments described above with an $h$-dipole pulse. 
The fields of an $h$-dipole pulse are written in terms of a function $\boldsymbol{Z}$ defined by

$$
\boldsymbol{Z}=\hat{\boldsymbol{z}} \frac{\mathrm{d}}{|\boldsymbol{x}|}[g(t+|\boldsymbol{x}|)-g(t-|\boldsymbol{x}|)],
$$

where the 'driving function' $g$ will be specified shortly and the 'virtual dipole moment' $d$ is a constant. The fields are

$$
B=-\nabla \times \nabla \times Z, \quad E=\nabla \times \dot{Z},
$$

and in the focus are equal to

$$
\boldsymbol{B}(0, t)=\hat{z} \frac{4 d}{3} \dddot{g}(t), \quad \boldsymbol{E}(0, t)=0 .
$$

For the driving function we choose a Gaussian,

$$
g(t)=\mathrm{e}^{-\Delta \omega^{2} t^{2} / 4} \sin (\omega t)
$$

in which $\omega$ is the central frequency and $\Delta \omega$ is a frequency spread. In the focus we have, from $(3.3 a, b)$, the same frequency spread as in $g$. The intensity distribution of the dipole pulse has the form (Gonoskov et al. 2012)

$$
I=I_{0} \sin ^{2} \theta,
$$

where $\theta$ is the angle made with the $z$-axis.

We will compare the flip probability in a dipole pulse with that in a Gaussian (paraxial) beam of the same input energy, using the expected parameters for the petawatt (PW) laser at DESY in conjunction with birefringence experiments (Schlenvoigt et al. 2016). We take a total energy of $30 \mathrm{~J}$, wavelength $\lambda=800 \mathrm{~nm}$ and a bandwidth of $\Delta \omega \simeq 0.035 \omega$ (corresponding to a FWHM pulse duration $28 \mathrm{fs}$ ). For the Gaussian we also need to choose a focal spot radius, which we take to be $w_{0}=1.75 \mu \mathrm{m}$, again following Schlenvoigt et al. (2016).

For these parameters the peak fields in the foci of the dipole and Gaussian beams become

$$
\frac{1}{2}\left(\boldsymbol{E}^{2}+\boldsymbol{B}^{2}\right) \simeq \begin{cases}3 \times 10^{-6} E_{S}^{2} & \text { Dipole } \\ 9 \times 10^{-8} E_{S}^{2} & \text { Gaussian }\end{cases}
$$

differing by over an order of magnitude: we may therefore expect a significantly stronger birefringence signal in the dipole pulse than in the Gaussian beam. However other factors also play a roll, e.g. polarisation alignment between probe and target (the dipole pulse is radially polarised). Turning to the flip probability (2.4) or (2.5) we also need a probe photon trajectory. We consider the best possible scenario where the probe passes through the field focus (i.e. zero impact parameter) at the instant of peak field strength (i.e. no timing miss). The resulting probabilities (naturally) follow the intensity profiles of the background fields. That in the dipole, for example, follows (3.5) and has the form

$$
\left|\mathbb{T}_{\text {flip }}\right|^{2}=C_{0} \sin ^{4} \theta
$$

where the incoming probe makes an angle $\theta$ to the $z$-axis and $C_{0}$ depends on the dipole pulse parameters and probe frequency, but not on $\theta$ or polarisation angles. 
(a)

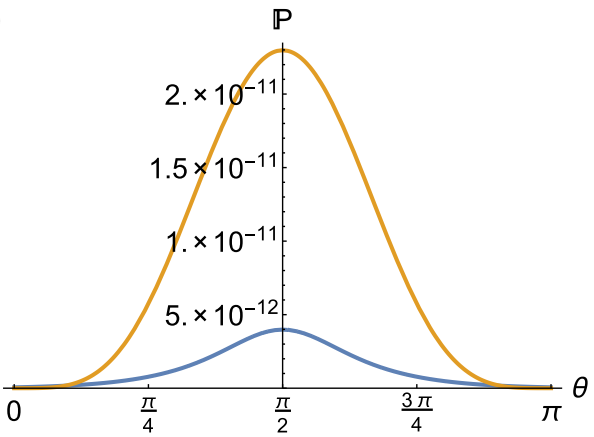

(b)

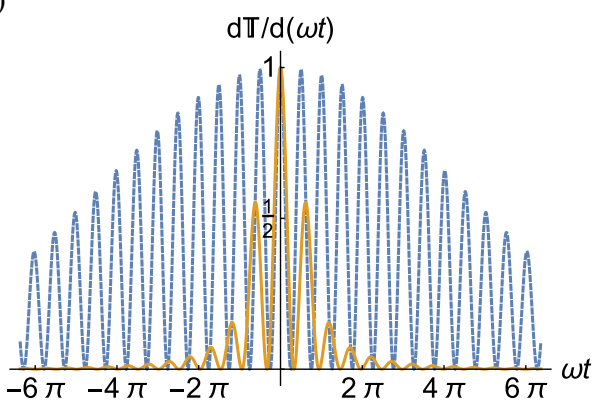

FIGURE 1. (a) Helicity-flip probability $\mathbb{P}$ in the dipole (yellow) and Gaussian (blue, with $\theta$ shifted by $\pi / 2$ to be able to plot on the same scale). (b) The integrands of the normalised probability amplitudes $\mathbb{T}$; in the Gaussian the focal spot is larger, and it can be seen that $\mathbb{T}$ indeed receives contributions from a larger time interval.

Further explicit expressions are unrevealing - instead we plot in figure 1 the flip probability in the dipole pulse and in the Gaussian beam. The probability in the dipole pulse exceeds that in the Gaussian by a factor of approximately 5.8, which is less than might be expected from (3.6). The reason for this is seen by recalling that it is an integrated parameter to which birefringence is sensitive, and that while the focal field strength in a dipole pulse is higher than in a Gaussian, the spot size is smaller. We can confirm this by estimating the effective transverse extent of the focus in our dipole pulse (transverse since the best case scenario is for probe angle $\theta=\pi / 2$ ). Following Gonoskov et al. (2012) we define the effective extent as the distance from the focal point at which the energy density drops to half its peak value. For our parameters we find a sub-wavelength extent $\simeq 0.4 \lambda$. In figure $1(a)$, we plot the (normalised) integrands of $\mathbb{T}$ in the dipole and Gaussian beams. We clearly see that the probability amplitude receives contributions from a much larger phase range in a Gaussian beam than it does in a dipole pulse; for the dipole case the width of the central peak is roughly $0.4 \lambda$, consistent with expectations.

\section{Synchrotron emission as a probe}

Above we discussed an 'alternative target' for measuring vacuum birefringence. We now turn to an 'alternative probe', namely synchrotron emission. We begin by recalling some standard results (Duke 2000). The spectral density of synchrotron emission from a particle with gamma factor $\gamma$ moving in planar circular motion, radius $R$, is

$$
I=I_{0} \gamma^{2}\left(\frac{\omega}{\omega_{c}}\right)^{2}\left(1+\gamma^{2} \psi^{2}\right)^{2}\left(K_{2 / 3}^{2}(\xi)+\frac{\gamma^{2} \psi^{2}}{1+\gamma^{2} \psi^{2}} K_{1 / 3}^{2}(\xi)\right),
$$

where the critical frequency is $\omega_{c}=3 \gamma^{3} / 2 R, \psi$ is the angle of elevation out of the plane of motion, $\xi=\left(\omega / \omega_{c}\right)\left(1+\gamma^{2} \psi^{2}\right)^{3 / 2}$ and $I_{0}$ is an overall normalisation which is not important here. The two terms in the large brackets of (4.1) represent, respectively, the intensities radiated parallel and perpendicular to the plane of motion, which we write as $I_{\|}$and $I_{\perp}$. Synchrotron radiation is highly plane polarised, as illustrated in figure 2. The small-angle part of the spectrum is therefore a potential source of highly 
(a)

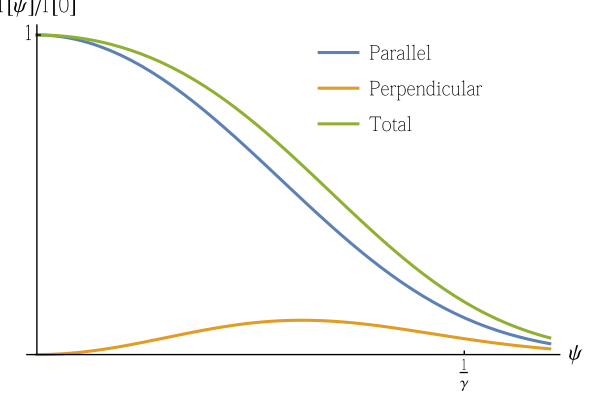

(b)

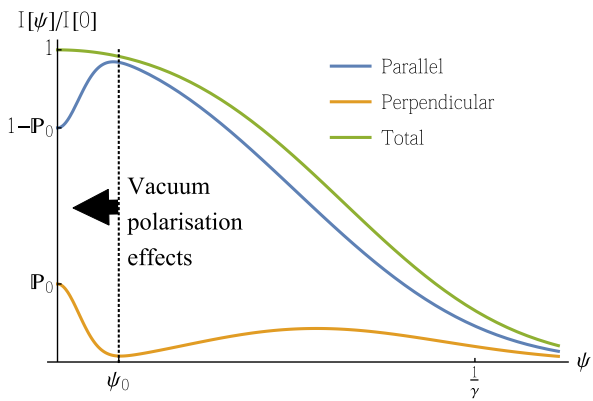

FIGURE 2. (a) Standard synchrotron emission spectrum as a function of opening angle $\psi$ at, to illustrate, $\omega^{\prime}=1.3 \omega_{c}$. The radiation is emitted in a narrow cone of opening angle $\psi \sim 1 / \gamma$. (b) The same spectrum illustrating the effects of vacuum polarisation, which are confined to narrower angles $\psi \lesssim \psi_{0}$ defined by the interaction geometry. Photon helicity flip mixes the parallel and perpendicularly polarised components of the synchrotron spectrum.

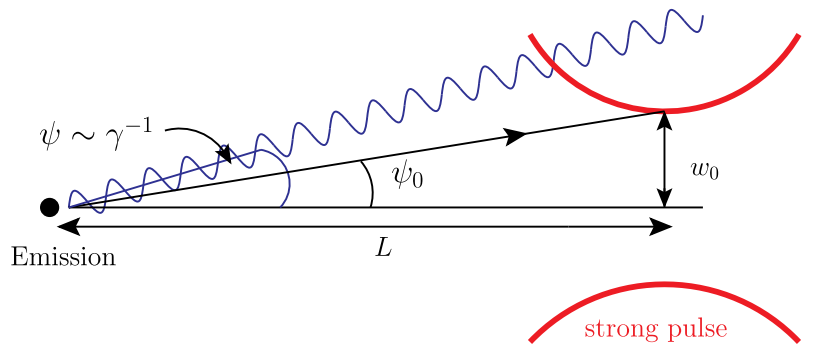

FIGURE 3. Sketch of experimental geometry, showing the distance between photon emission and interaction points. Emission is near forward, $\psi<1 / \gamma$, while the effective emission range of photons which can interact with the high-intensity pulse is limited to $\psi<\psi_{0}:=\tan ^{-1} L / w_{0}$.

polarised photons for use in birefringence experiments: if these photons interact with an intense optical pulse, helicity flip will mix the plane and perpendicularly polarised parts of the emitted radiation, 'deforming' the synchrotron spectrum. (Of course we need a high-energy synchrotron spectrum to obtain an appreciable flip probability, see below.)

The portion of photons which will interact with the focal spot of the intense pulse is limited by the geometry of the experiment. Assume that the distance between the emission point of the high-energy photons and the focal point of the high-intensity pulse is $L$, and that the pulse's focal width is $w_{0}-$ see figure 3 . Clearly only photons emitted in a very narrow angle $\psi<\psi_{0}:=\tan ^{-1} w_{0} / L$ will see the laser focal spot and be likely to change helicity state*. This will be, as we verify below, much smaller than the typical opening angle $(1 / \gamma)$ of the synchrotron spectrum, so vacuum polarisation effects will only be observable for photons emitted almost within the plane of motion of the electrons.

Given a set-up as in figure 3 we can use (2.4) to calculate the flip probability $\mathbb{P}$. Let $\mathbb{P}_{0}$ be the 'best case' flip probability for photons arriving at the focal spot at the

*We consider only photons which arrive at the focal spot at the instance of peak field strength - for the impact of timing jitter, see Dinu et al. (2014a) and Schlenvoigt et al. (2016). 
instant of peak field strength and with polarisation at $45^{\circ}$ to that of the intense optical pulse. Now consider deviations from the ideal case: the dependence of the probability on emission angle $\psi$ is easily guessed as being Gaussian, since the probability is expected to follow the intensity distribution squared. Indeed

$$
\mathbb{P}\left(\omega^{\prime}, \psi\right)=\mathbb{P}_{0}\left(\omega^{\prime}\right) \mathrm{e}^{-4 \psi^{2} / \psi_{0}^{2}},
$$

gives a very good approximation of the flip probability: additional dependencies on geometric or polarisation angles due to perturbing away from the ideal case are effectively washed out by the very rapid falloff of the probability with $\psi$. With this we can write down a simple model of the synchrotron emission spectrum following interaction with an intense pulse. Denoting the outgoing distribution with a prime, we write

$$
\left.\begin{array}{l}
I_{\|}^{\prime}=(1-\mathbb{P}) I_{\|}+\mathbb{P} I_{\perp}, \\
I_{\perp}^{\prime}=(1-\mathbb{P}) I_{\perp}+\mathbb{P} I_{\|},
\end{array}\right\}
$$

(implying no photons are lost: $I^{\prime}:=I_{\|}^{\prime}+I_{\perp}^{\prime}=I$ ). Vacuum polarisation then has a significant impact on the spectrum only for $\psi<\psi_{0}$, as is illustrated in figure $2(b)$. With this model in hand we turn to quantitative estimates for a proposed experiment at ELI-NP (Nakamiya et al. 2015).

\subsection{Experiments at ELI-NP}

The ELI proposal begins with the collision of highly relativistic electrons, $\gamma \gg 1$, with a linearly polarised laser pulse. The electrons undergo Compton back scattering and emit instantaneously in a synchrotron spectrum (Jackson 1998). The polarisation direction of the emitted radiation is set by the plane of motion of the electrons, which is in turn set by the laser polarisation direction. The angle $\psi$, above, is the angle out of this plane.

The produced high-energy photons are then used as the probe of a (second) laser pulse of very high intensity. The combination of high intensity and high energy increases the probability of helicity flip as the probe photons pass through the optical laser, see (2.4)-(2.5). After this interaction the polarisation of the high-energy photons is measured using a pair polarimeter (Nakamiya et al. 2015), see also below.

We assume generation of $2 \mathrm{GeV}$ electrons which are collided with a laser pulse of, by modern standards, moderate intensity $I \sim 10^{20} \mathrm{~W} \mathrm{~cm} \mathrm{~cm}^{-2}$. This generates radiation with a critical frequency of $\omega_{c}=(3 / 2) m \gamma^{2}\left(E / E_{S}\right)=0.24 \mathrm{GeV}$ (using $I=E^{2} / 2$ ) which is to interact with an intense optical pulse.

We assume a distance of $L=20 \mathrm{~cm}$ between the emission point of the radiation and the interaction point with the intense pulse. Based on expected ELI parameters we take a focal radius $w_{0}=2.5 \mu \mathrm{m}$. This gives the effective emission angle as $\psi_{0}=\tan ^{-1} 2.5 \mu \mathrm{m} / 20 \mathrm{~cm} \simeq 10^{-5}$, which is as suggested above much less than $1 / \gamma \sim 3 \times 10^{-4}$. In order to write down the flip probability, we need the 'best case' result as described above (4.2). For a focussed Gaussian beam this has been found in Dinu et al. $(2014 a)$ to be

$$
\mathbb{P}_{0}\left(\omega^{\prime}\right)=\left(\frac{\alpha}{15} \frac{1}{E_{S}^{2}} \frac{\mathcal{E} \omega^{\prime}}{\pi^{2} w_{0}^{2}}\right)^{2},
$$

where $\mathcal{E}$ is the energy of the laser. Based on expected ELI parameters we take $\mathcal{E}=200 \mathrm{~J}$, which gives

$$
\mathbb{P}_{0}\left(\omega^{\prime}\right) \simeq 0.27\left(\frac{\omega^{\prime}}{\mathrm{GeV}}\right)^{2}
$$




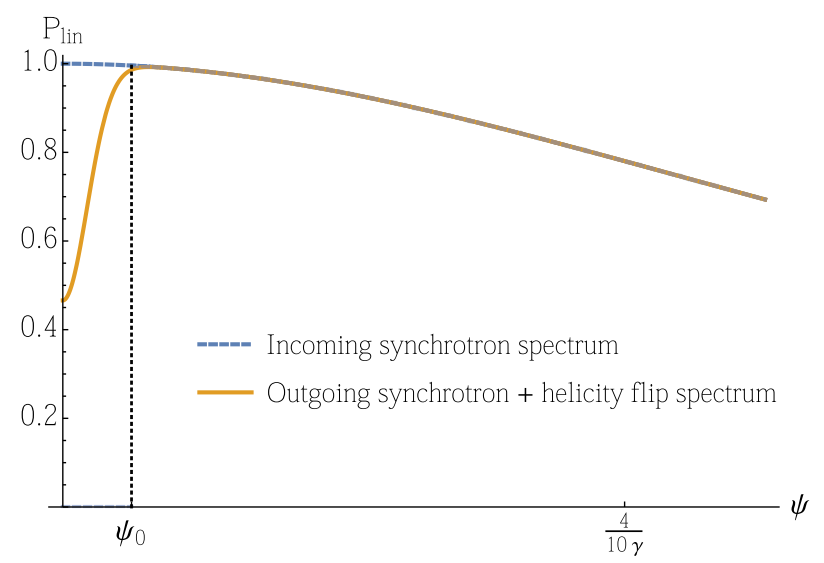

FIGURE 4. The degree of linear polarisation in the synchrotron spectrum (blue/dashed) and in the spectrum after passing through an intense field (yellow/solid) in which vacuum polarisation effects cause changes in photon polarisation. Plotted for $1 \mathrm{GeV}$ probe photon energy and other parameters as in the text, for the proposed set-up at ELI-NP.

This is significantly higher than for an optical X-ray set-up simply due to the higher probe energy (and the expected higher energies and intensities available at ELI). The flip probability as a function of $\psi$ is then given by (4.2).

In the proposed experiment, the emitted radiation will be screened in order to ensure a high polarisation purity; only that part of the spectrum emitted at $\psi$ less than some small fraction, say $40 \%$, of $1 / \gamma$ will be allowed to propagate toward the high-intensity pulse (the target). A detector will be arranged to screen out (to some high degree) plane-polarised emission. The signal to be measured is then the increase in perpendicularly polarised photons incident on the detector due to vacuum polarisation effects. We can calculate the total energy deposited on the detector due to perpendicularly polarised photons from $^{\dagger}$ (Jackson 1998)

$$
\mathrm{En}_{\perp}^{\prime}=\int_{0}^{0.4 / \gamma} \mathrm{d} \psi \int_{0}^{2 \mathrm{GeV}} \mathrm{d} \omega^{\prime} I_{\perp}^{\prime}\left(\omega^{\prime}, \psi\right) \simeq 1.16 \mathrm{En}_{\perp},
$$

which gives an increase of $16 \%$. A convenient measure of polarisation purity (which is also related to the polarimetry required to measure the photon polarisation in this set-up (Nakamiya et al. 2015)) is the 'degree of linear polarisation', which for the synchrotron spectrum before and after interaction with the intense laser pulse is defined by

$$
P_{\text {lin }}:=\frac{I_{\|}-I_{\perp}}{I_{\|}+I_{\perp}}, \quad P_{\text {lin }}^{\prime}=\frac{I_{\|}^{\prime}-I_{\perp}^{\prime}}{I_{\|}^{\prime}+I_{\perp}^{\prime}}=(1-2 \mathbb{P}) P_{\text {lin }} .
$$

Vacuum polarisation effects will (for $\mathbb{P}<0.5$ ) reduce the degree of linear polarisation: this is illustrated for the parameters considered here in figure 4.

\footnotetext{
†We integrate only up the electron energy, as quantum effects will cutoff the spectrum there: this and other such refinements should be included in future calculations.
} 


\section{Discussion and conclusions}

We have considered two proposals for measuring vacuum polarisation effects in strong laser fields. In the first, we used a dipole pulse as the 'target'. The optimal focussing of dipole pulses yields high focal field strengths, which makes them ideal for studying pair creation (Gonoskov et al. 2013) and intense-field dynamics (Gonoskov et al. 2014). However the dipole pulse has a small (subwavelength) focal spot size, which can be disadvantageous for vacuum birefringence as the relevant observable there is sensitive to, essentially, the product of field strength and spot size.

The second method we have considered is the use of laser-particle collisions to generate high-energy gamma rays, which are in turn used as the probe of an intense optical pulse. We have provided a very simple 'proof of principle' calculation and seen that the high energy of the probe photons gives a large (ideal case) helicityflip probability. The experimental realisation of the relevant set-up though will be challenging. Measurement of the signal requires pair polarimetry on the probe gamma rays; this is discussed in Nakamiya et al. (2015). The use of a laser-particle collision to generate the probe in close proximity to the target suggests a 'messy' experimental environment. Only photons generated in a small volume of space, at the right time, will interact with the focal spot of the intense pulse and have an appreciable chance of changing helicity state; however the actual generation point can be anywhere in the volume of the laser-particle collision. This suggests that shot-shot fluctuations in signal and background may be large.

Refinements of the calculation presented here could begin with numerical simulations of the initial laser-particle interaction in order to better understand the spectrum of the generated probe photons (Elkina \& King 2016). Here PIC methods would be useful, for a review of which, see Gonoskov et al. (2015). (Polarisation effects would of course need to be included.) Once the spectrum is understood the impact of effects such as timing and pointing jitter can be included, and then a comprehensive picture of the background and signal sizes can be developed.

\section{Acknowledgements}

The authors are supported by the Olle Engkvist Foundation, grant 2014/744 (A.I.) and the Swedish Research Council, grants 2012-5644 and 2013-4248 (M.M.). A.I. thanks the coordinators of ELI-NP for the opportunity to join the facility's Technical Design Report.

\section{REFERENCES}

ELI 2014 Technical design report of the extreme light infrastructure - nuclear physics facility, Romania. http://www.eli-np.ro/.

HIBEF 2015 Helmholtz international beamline for extreme fields at the European XFEL. http://www.hzdr.de/db/Cms?pOid=35325\&pNid=3214.

Di Piazza, A., Hatsagortsyan, K. Z. \& Keitel, C. H. 2006 Light diffraction by a strong standing electromagnetic wave. Phys. Rev. Lett. 97, 083603.

Dinu, V., Heinzl, T., Ilderton, A., Marklund, M. \& Torgrimsson, G. 2014a Photon polarization in light-by-light scattering: finite size effects. Phys. Rev. D 90 (4), 045025.

Dinu, V., Heinzl, T., Ilderton, A., Marklund, M. \& Torgrimsson, G. 2014b Vacuum refractive indices and helicity flip in strong-field QED. Phys. Rev. D 89 (12), 125003.

Duke, P. J. 2000 Synchrotron Radiation: Production and Properties. University Press. 
ElKINA, N. \& KING, B. 2016 Vacuum birefringence in high-energy laser-electron collisions (to appear).

Euler, H. \& Kockel, B. 1935 Ueber die Streuung von Licht an Licht nach der Diracschen Theorie. Naturwissenschaft 23, 246.

Gies, H., Karbstein, F. \& Seegert, N. 2013 Quantum reflection as a new signature of quantum vacuum nonlinearity. New J. Phys. 15, 083002.

Gies, H., Karbstein, F. \& Seegert, N. 2015 Quantum reflection of photons off spatio-temporal electromagnetic field inhomogeneities. New J. Phys. 17 (4), 043060.

Gonoskov, A., Bashinov, A., Gonoskov, I., Harvey, C., Ilderton, A., Kim, A., Marklund, M., Mourou, G. \& Sergeev, A. 2014 Anomalous radiative trapping in laser fields of extreme intensity. Phys. Rev. Lett. 113, 014801.

Gonoskov, A., Bastrakov, S., Efimenko, E., Ilderton, A., Marklund, M., Meyerov, I., Muraviev, A., Sergeev, A., Surmin, I. \& Wallin, E. 2015 Extended particle-in-cell schemes for physics in ultrastrong laser fields: review and developments. Phys. Rev. E 92 (2), 023305.

Gonoskov, A., Gonoskov, I., Harvey, C., Ilderton, A., Kim, A., Marklund, M., Mourou, G. \& SERgeEV, A. M. 2013 Probing nonperturbative QED with optimally focused laser pulses. Phys. Rev. Lett. 111, 060404.

Gonoskov, I., Aiello, A., Heugel, S. \& Leuchs, G. 2012 Dipole pulse theory: maximizing the field amplitude from $4 \pi$ focused laser pulses. Phys. Rev. A 86, 053836.

HALPERN, O. 1933 Scattering processes produced by electrons in negative energy states. Phys. Rev. 44, 855-856.

Heinzl, T., Liesfeld, B., Amthor, K-U., Schwoerer, H., SAuerbrey, R. \& Wipf, A. 2006 On the observation of vacuum birefringence. Opt. Commun. 267, 318-321.

HeisenberG, W. \& Euler, H. 1936 Consequences of Dirac's theory of positrons. Z. Phys. 98, 714-732.

JACKSON, J. D. 1998 Classical Electrodynamics. Wiley.

KARbSTEIN, F. 2015 Vacuum birefringence as a vacuum emission process. In Photon 2015: International Conference on the Structure and Interactions of the Photon and the 21th International Workshop on Photon-Photon Collisions and International Workshop on High Energy Photon Linear Colliders Novosibirsk, Russia, June 15-19, 2015.

Karbstein, F., Gies, H., Reuter, M. \& Zepf, M. 2015 Vacuum birefringence in strong inhomogeneous electromagnetic fields. Phys. Rev. D 92 (7), 071301.

KIM, J. Y. \& LEE, T. 2011 Light bending by nonlinear electrodynamics under strong electric and magnetic field. JCAP 1111, 017.

King, B., Di PiazzA, A. \& Keitel, C. H. 2010 A matterless double slit. Nature Photon. 4, 92-94.

KING, B. \& HEINZL, T. 2015 Measuring vacuum polarisation with high power lasers; arXiv: 1510.08456.

King, B. \& Keitel, C. H. 2012 Photon-photon scattering in collisions of laser pulses. New J. Phys. 14, 103002

Lundstrom, E., Brodin, G., Lundin, J., Marklund, M., Bingham, R., Collier, J., Mendonca, J. T. \& Norreys, P. 2006 Using high-power lasers for detection of elastic photon-photon scattering. Phys. Rev. Lett. 96, 083602.

Marklund, M. \& Shukla, Padma Kant 2006 Nonlinear collective effects in photon-photon and photon-plasma interactions. Rev. Mod. Phys. 78, 591-640.

NakamiYa, Y., Homma, K., Moritaka, T. \& Seto, K. 2015 Probing vacuum birefringence under a high-intensity laser field with gamma-ray polarimetry at the GeV scale; arXiv:1512.00636.

NArozhny, N. B. 1969 Propagation of plane electromagnetic waves in a constant field. Sov. Phys. JETP 28 (2), 371-374.

Schlenvoigt, H-P., Heinzl, T., Schramm, S., Cowan, T. E. \& Sauerbrey, R. 2016 Detecting vacuum birefringence with X-ray free electron lasers and high-power optical lasers: a feasibility study. Phys. Scr. 91 (2), 023010.

Toll, J. S. 1952 The Dispersion relation for light and its application to problems involving electron pairs. PhD thesis, Princeton University.

Torgrimsson, G. 2015 Ellipticity induced in vacuum birefringence. Few Body Syst. 56 (6-9), $615-620$. 